\title{
Restauração de serviços em nuvem óptica: uma abordagem tolerante à degradação de banda e ao atraso de restauração
}

\author{
Felipe Lisboa ${ }^{1}$, Keiko V. O. Fonseca ${ }^{1}$ (coorientadora), \\ Juliana de Santi $^{1}$ (orientadora) \\ ${ }^{1}$ Universidade Tecnológica Federal do Paraná (UTFPR) - Curitiba \\ felipe@ecentric.com.br, \{keiko,jsanti\}@utfpr.edu.br
}

\begin{abstract}
This paper address the problem of restoration of optical cloud services in the presence of a single fiber link failure. The proposed algorithm, named $R 3 D$, leverages on two parameters specified in the service class (restoration delay and bandwidth degradation) in order to make the best use of the available optical resources during the recovery process. Results, derived via simulation, show significant improvements on cloud service restorability without a negative impact on the cloud service blocking probability.
\end{abstract}

Resumo. Este artigo, aborda o problema de restauração de serviços na nuvem com infraestrutura óptica na presença de falha em um único enlace. $O$ algoritmo proposto, denominado R3D, leva em consideração dois parâmetros especificados em classes de serviço (tolerância ao atraso na restauração $e$ degradação de banda passante) para fazer melhor uso dos recursos ópticos disponiveis durante o processo de restauração. Resultados, obtidos através de simulação, demonstram melhorias significativas na capacidade de restauração de serviços sem impactar de forma negativa a probabilidade de bloqueio.

\section{Introdução}

Redes ópticas são soluções atraentes para suportar uma ampla gama de serviços na nuvem tais como aplicações científicas, de negócios e para o consumidor (por exemplo, serviços de entrega de conteúdo). A grande disponibilidade de banda passante, baixa latência e eficiência energética são algumas das características que fazem as redes ópticas adequadas para estas tarefas [Kachris and Tomkos 2012]. No entanto, devido a cortes acidentais de fibras, falhas de equipamentos ou mesmo ataques maliciosos, a infraestrutura óptica é suscetível a falhas. Na presença de falhas, um ou mais caminhos ópticos podem ser interrompidos, afetando vários serviços na nuvem e causando, consequentemente, a perda de grande quantidade de dados. Devido ao papel crucial dos serviços em nuvem em nossa sociedade, estratégias para a recuperação de falhas precisam ser desenvolvidas para manter um nível aceitável de sobrevivência de serviços enquanto asseguram que a resiliência seja provida com um custo extra contido em relação à eficiência na utilização dos recursos ópticos.

Estratégias de recuperação de falhas baseadas em proteção alocam recursos ópticos redundantes, os quais são usados somente na ocorrência da falha. Como resultado, estas estratégias garantem $100 \%$ de recuperação, mas têm custo elevado em termos de eficiência na utilização dos recursos, pois os recursos para proteção não são utilizados na maior parte do tempo [da Silva et al. 2016]. Para reduzir tais custos, as operadoras podem usar estratégias baseadas no conceito de restauração. Nesta abordagem não 
há reserva antecipada de recursos ópticos. Após a ocorrência de uma falha, os caminhos afetados são reroteados baseando-se somente nos recursos disponíveis pós-falha, de forma a restaurar o máximo possível de serviços na nuvem. Assim, as estratégias de restauração são mais eficientes no uso de recursos, mas não podem garantir $100 \%$ de recuperação [da Silva et al. 2016].

A literatura apresenta vários estudos envolvendo estratégias de restauração. Alguns deles levam em consideração a degradação de serviço, que é definida a partir de requisitos de serviços especificados em contratos de serviço SLA (Service Level Agreements). Uma possibilidade é a degradação de banda passante durante o processo de restauração [Huang et al. 2010, Wang et al. 2015, Bao et al. 2016]. Outra opção é tirar vantagem da informação sobre o atraso máximo permitido para a restauração (ou seja, o tempo entre a ocorrência da falha e o tempo no qual o serviço é restaurado) para determinar o momento da restauração do serviço na nuvem [Khabbaz and Assi 2015, Ferdousi et al. 2015, Wang et al. 2016]. Embora as direções de pesquisa mencionadas tenham sido extensivamente estudadas, o uso combinado de degradação de banda passante e atraso na restauração não tem sido investigado.

O presente artigo descreve um novo algoritmo de restauração de serviços, RD3, desenvolvido como trabalho de Iniciação Científica, para nuvens com infraestrutura óptica. O diferencial do algoritmo proposto é tirar vantagem da utilização conjunta da tolerância ao atraso na restauração e da degradação de banda passante para oferecer uma estratégia que seja eficiente na utilização de recursos para a recuperação de serviços em nuvens ópticas. A combinação destes conceitos pode ser particularmente útil uma vez que serviços na nuvem têm diferentes requisitos [Develder et al. 2012], os quais podem ser arranjados de forma estratégica para a recuperação destes serviços. Aplicações hardreal-time (por exemplo, procedimentos cirúrgicos) devem ser imediatamente restauradas usando um caminho óptico alternativo com capacidade suficiente para recuperar completamente o serviço na nuvem. Alternativamente, aplicações soft-real-time (por exemplo, video streaming) podem ser restauradas em um caminho óptico com capacidade reduzida, degradando-se o serviço para um nível mínimo aceitável. Por sua vez, aplicações nonreal-time (por exemplo, serviços em grade para processamento de dados) são flexíveis tanto no domínio de banda quanto no domínio de tempo. Por exemplo, a restauração destes serviços pode ser postergada com base na sua tolerância ao atraso na recuperação. Ao atrasar a recuperação de um serviço non-real-time, os recursos ópticos disponíveis podem ser usados para a restauração de serviços críticos, ou seja, hard-real-time. Além disso, a capacidade extra economizada (ou seja, ao permitir a degradação de banda de algum serviço durante o processo de recuperação) pode ser alocada para restaurar serviços adicionais que de outra forma seriam perdidos. Consequentemente, é possível aumentar o número total de serviços restaurados na nuvem.

$\mathrm{Na}$ heurística proposta, após um evento de falha, serviços do tipo hard e soft-realtime são restaurados imediatamente (com banda total ou parcial) não tendo que competir por recursos com serviços que podem ser restaurados em algum momento posterior. Serviços non-real-time, por sua vez, podem se beneficiar da redução de banda bem como de um tempo de restauração mais longo para prevenir a perda destes serviços. Resultados derivados via simulação indicam que o algoritmo proposto é capaz de aumentar o número de serviços restaurados na nuvem óptica sem impactar de forma negativa a probabilidade 
de bloqueio na rede. Estes resultados foram relatados em [Lisboa et al. 2017], publicado na conferência internacional "IEEE Global Communications Conference" (GLOBECOM), Qualis A1.

Este trabalho está organizado conforme segue. A Seção 2 apresenta os trabalhos correlatos. A Seção 3 introduz o algoritmo proposto R3D. Na Seção 4, apresenta-se a avaliação numérica do algoritmo proposto. Na Seção 5 são delineadas as conclusões. A Seção 6 apresenta a síntese das principais contribuições do trabalho de Iniciação Científica.

\section{Trabalhos relacionados}

O impacto gerado por interrupções na infraestrutura da rede tem motivado o estudo de estratégias para recuperação rápida e eficiente de falhas na rede. Tais estratégias podem ser projetadas considerando as diferentes características e requisitos dos serviços. Os autores em [Develder et al. 2012] apresentam uma classificação para aplicações científicas, de negócios e para consumidores considerando seus requisitos na nuvem suportada por redes ópticas. Eles especificam a sensibilidade ao atraso de acordo com a classificação das aplicações.

Existe vasta literatura relacionada à recuperação de falhas baseada em proteção [Habib et al. 2013, Natalino et al. 2015], que garante a recuperação através da redundância de recursos. Uma abordagem de menor custo mas ainda eficiente é a restauração de serviços, a qual tenta recuperar os serviços usando caminhos ópticos alternativos de forma reativa à falhas [Huang et al. 2010, Wang et al. 2015, da Silva et al. 2016]. O conceito de degradação de serviços, que garante uma quantidade parcial da banda passante originalmente requisitada pela aplicação, foi usado na investigação apresentada em [Huang et al. 2010] no uso estratégias para o provisionamento resiliente de serviços. Considerando cenários de desastres, o trabalho em [Bao et al. 2016] descreve uma estratégia de re-provisionamento de serviços baseada em degradação de banda e roteamento multi-caminho para manter a conectividade da rede e balancear a distribuição de tráfego. Os autores em [Wang et al. 2015] apresentam um modelo de programação linear inteira (PLI) baseado na degradação de serviços e realocação para restauração de serviços em nuvem (a realocação consiste em migrar os serviços de um nó Data Center (DC) que falhou para um nó DC alternativo ativo). Ademais, visando minimizar o número de serviços perdidos e a quantidade de recursos usada na restauração, um serviço restaurado é provisionado usando somente a metade da capacidade de um comprimento de onda. A estratégia de restauração apresentada em [da Silva et al. 2016] combina os benefícios da realocação e diferenciação de serviços de forma a melhorar a capacidade de restauração enquanto garante que diferentes serviços recebem prioridades apropriadas.

O uso de informações de prazo (deadline) para a tomada de decisão em sistemas envolvendo nuvens e redes ópticas é um campo ativo de pesquisa [Wang et al. 2012, Khabbaz and Assi 2015, Ferdousi et al. 2015, Wang et al. 2016, Bao et al. 2016]. Os autores em [Khabbaz and Assi 2015] apresentam um modelo analítico de fila para escalonamento flexível de tarefas com especificações de tempo em nuvens. Os autores em [Wang et al. 2012] consideram que o estabelecimento de conexões inter-DC são tolerantes ao atraso e que a banda liberada durante o período de espera pode ser 
usada para completar a transferência de dados de conexões postergadas. Considerando informações de alerta de desastres e prazo para evacuação de dados, os autores em [Ferdousi et al. 2015] apresentam uma heurística pró-ativa para evacuar conteúdo vulnerável e crítico de prováveis zonas de desastres para localizações seguras através da utilização de caminhos ópticos que maximizam a quantidade de dados evacuados. Eles classificam a vulnerabilidade do conteúdo baseado na presença da réplicas DC mais atualizadas em zonas de desastres.

Diferentemente das estratégias de recuperação de falha baseadas em restauração, o algoritmo proposto neste trabalho explora a flexibilidade resultante da combinação de degradação de banda passante e atraso na restauração de forma a aumentar as chances de restauração de serviços em redes de nuvem óptica. Os requisitos de serviços são especificados considerando diferentes classes de serviços.

\section{Algoritmo proposto}

O algoritmo descrito nesta seção, denominado Restoration of Differentiated Cloud Services based on Bandwidth Degradation and Restoration Delay (R3D), tira vantagem de diferentes requisitos de serviço para recuperar serviços interrompidos por uma falha através da restauração na nuvem com degradação de banda e diferentes níveis de tolerância ao atraso na recuperação. $\mathrm{O}$ atraso na restauração ocorre somente para serviços non-realtime, enquanto a degradação de banda pode ser usada para serviços non-real-time e softreal-time. Além disso, o nível de degradação de serviço é estritamente aquele definido pelo SLA de cada serviço.

A seguir são descritos o modelo de estabelecimento de serviço, as classes de serviço usadas e a descrição formal do algoritmo proposto.

\subsection{Estabelecimento de solicitações de serviço}

No cenário considerado neste trabalho, os nós clientes solicitam serviços na nuvem compostos por unidades para armazenamento e para processamento, as quais são disponibilizadas em nós Data Center (DC) geograficamente distribuídos. Uma rede óptica (WDM - Wavelength Division Multiplex ou EON - Elastic Optical Network) interconecta os nós clientes aos nós DCs. Além disso, solicitações de serviço são classificadas em Classes de Serviço $(\mathrm{CoS})$ de acordo com a sua tolerância para degradação de banda passante e sua tolerância para atraso na restauração (Seção 3.2). Note que esta classificação é levada em consideração somente para a restauração de serviços interrompidos, não tendo influência no provisionamento dos serviços na nuvem óptica.

Uma requisição de serviço $r(b, h, v m, s t, c l, A R)$, que chega a rede de forma dinâmica, especifica a banda demandada $(b)$, o tempo de duração $(h)$, o número de maquinas virtuais $(\mathrm{vm})$ e o número de unidade de armazenamento $(\mathrm{st})$, sua classe de serviço $(c l)$ bem como seu nível de tolerância para atraso na restauração $(A R)$. Então, o nó DC mais próximo, com maquinas virtuais e unidades de armazenamento suficiente, e com recursos de banda passante suficientes ao longo da rota, é escolhido para suportar a conexão para o serviço. Caso não seja possível encontrar nó DC ou caminho óptico com recurso disponível, a solicitação para o serviço na nuvem é bloqueada. 


\subsection{Prioridades e requisitos de serviços na nuvem}

Em geral, após uma falha, a disponibilidade de recursos ópticos pode não ser suficiente para recuperar todos os serviços atingidos. Assim, ter uma política de restauração com diferenciação de serviços é essencial para aumentar o número de serviços restaurados e garantir os requisitos de SLA dos serviços recuperados. Considerando a classificação de serviços apresentada em [Develder et al. 2012], a Tabela 1 ilustra os requisitos e prioridades dos serviços considerados neste trabalho. A tabela apresenta três classes de serviço: hard-real-time, soft-real-time e non-real-time. Após a detecção de uma falha, os serviços da classe hard-real-time tem a maior prioridade no processo de restauração e precisam ser recuperados imediatamente (ou seja, não é permitido atraso na restauração) com a banda total requisitada (ou seja, não é permitida degradação em sua banda passante).

Tabela 1. Prioridade e requisitos de serviços na nuvem.

\begin{tabular}{cccc}
\hline Real-time & Prioridade & Degradação de banda & Atraso na restauração (AR) \\
\hline hard & alta & não aceitável & não aceitável \\
soft & média & metade & não aceitável \\
non & baixa & metade & aceitável \\
\hline
\end{tabular}

Os serviços soft-real-time são sensíveis ao atraso na recuperação, mas permitem a redução da metade de sua banda passante no processo de restauração [Wang et al. 2015]. Os serviços da classe non-real-time têm a menor prioridade e são recuperados somente após a restauração dos serviços de todas as outras classes. Adicionalmente, esta classe permite degradação de banda e atraso na restauração, sendo a classe mais flexível e, por isso, a que proporciona as maiores oportunidade a serem exploradas em nossa abordagem.

\subsection{Restauração de serviço na nuvem}

No algoritmo de restauração R3D (Algoritmo 1), os serviços são restaurados com base em suas prioridades, conforme descrito na Seção 3.2. A degradação de banda ocorre somente se não há disponibilidade de banda suficiente para a restauração total dos serviços softreal-time e non-real-time. Ademais, para serviços da classe non-real-time a restauração é atrasada somente após uma tentativa mal sucedida de degradação de banda.

O R3D é executado sempre que um conjunto $S(l)$ de serviços na nuvem são interrompidos devido à uma falha no enlace $l$. Na Linha 1 , o grafo auxiliar é construído com os recursos ópticos disponíveis para utilização no processo de restauração. Na Linha 2, os serviços em $S$ são ordenados em ordem decrescente de prioridade assegurando que os serviços hard-real-time tenham precedência sobre os serviços soft-real-time e non-realtime quando eles competem pelos mesmos recursos. Da mesma forma, serviços da classe soft-real-time têm precedência sobre os serviços non-real-time.

Para cada $s_{i}$ em $S$ (Linha 3), a classe a qual $s_{i}$ pertence é verificada de forma a garantir o procedimento de restauração apropriado (Linha 4, Linha 9 e Linha 17). Se $s_{i}$ é um serviço hard-real-time (Linha 4), não há tolerância para degradação de banda nem para atraso na restauração e, assim, um caminho $l p$ com pelo menos $b$ unidades de banda disponível deve existir (Linha 5) para que se possa restaurar $s_{i}$ usando $b$ unidades de banda (Linha 6). Caso contrário, os requisitos para o serviço $s_{i}$ não podem ser atendidos e este serviço hard-real-time é perdido (Linha 8). 


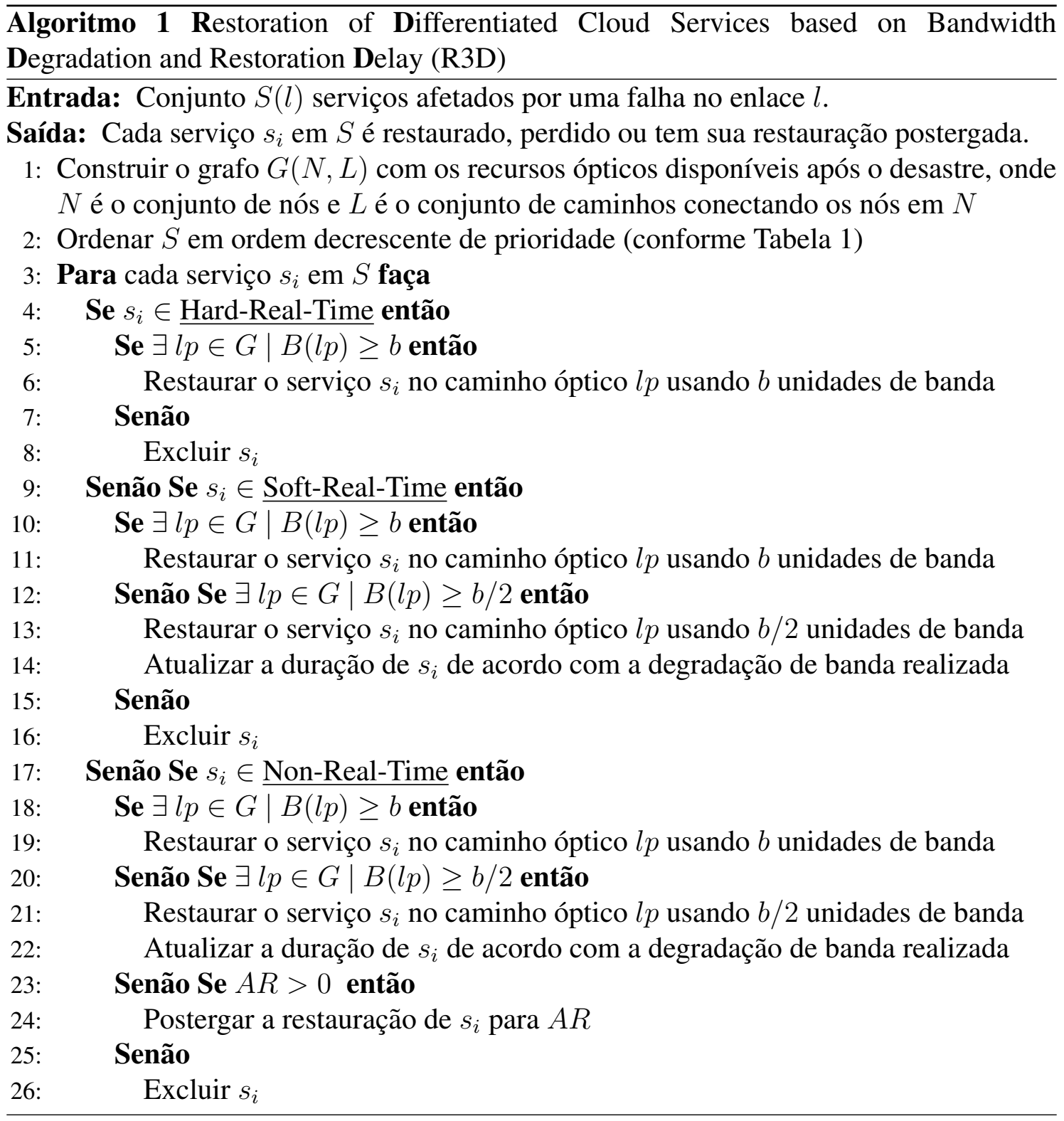

Se $s_{i}$ é um serviço soft-real-time (Linha 9) e existe um caminho óptico lp com pelo menos $b$ unidades de banda (Linha 10) $s_{i}$ é restaurado (Linha 11). Se este não é o caso, o algoritmo verifica a existência de um caminho óptico $l p$ com pelo menos a metade da banda requisitada (Linha 12) para restaurar $s_{i}$ com degradação de banda (Linha 13). Neste caso, há um aumento do tempo de duração do serviço (Linha 14) para compensar a redução na taxa de transmissão. Note que $s_{i}$ é degradado somente se a banda disponível é menor que a banda requisitada $(B<b)$. Além disso, $s_{i}$ pode ser degrado somente uma vez durante seu provisionamento. Se não há um caminho com pelo menos $b / 2$ unidades de banda, a restauração torna-se impraticável uma vez que não é possível garantir o requisito mínimo, e o serviço soft-real-time é perdido (Linha 16).

Se $s_{i}$ é um serviço non-real-time (Linha 17) existe flexibilidade para degradação de banda e para atraso na restauração. Em caso de disponibilidade de recursos (Linha 18), $s_{i}$ é restaurado usando a banda requisitada $b$ (Linha 19). Se há pelo menos a metade da 
banda requisitada (Linha 20), $s_{i}$ é restaurado com $b / 2$ unidades de banda (Linha 21) e a devida extensão na duração da transmissão é realizada (Linha 22). Se houver indisponibilidade de banda passante, mas existir alguma tolerância ao atraso na recuperação $A R$ (Linha 23), a restauração para o serviço $s_{i}$ é postergada e uma nova tentativa de restauração é escalonada de acordo com $A R$ (Linha 24). Note que a restauração após $A R$ unidades de tempo deve garantir a banda requisitada $b$ bem como o tempo de transmissão restante para o serviço. Além disso, um serviço non-real-time pode ser degradado somente uma vez durante seu provisionamento, e é perdido somente se não há banda disponível e o tempo de tolerância para atraso na restauração acabou (Linha 26).

\subsubsection{Complexidade computacional}

A construção do grafo $G$ envolve $\mathcal{O}\left(N^{2}\right)$ operações, onde $N$ é o número de nós na rede (Linha 1). Considerando que a menor taxa de transmissão é $X-O C$ (Optical Carrier) e a capacidade de um comprimento de onda é $Y-O C$, podem existir no máximo $Y / X$ serviços interrompidos no conjunto $S$, então existem $\mathcal{O}(Y / X)$ operações na Linha 3, onde $Y / X$ é uma constante. Embora os elementos do conjunto $S$ sejam ordenados (Linha 2), seu custo é, também, uma constante uma vez que há no máximo $Y / X$ serviços em $S$. Para encontrar o caminho com a banda solicitada, o algoritmo Dijkstra requer $\mathcal{O}\left(N^{2}\right)$ operações (Linha 5, Linha 10, Linha 12, Linha 18 e Linha 20). Assim, a complexidade do algoritmo R3D é $\mathcal{O}\left(N^{2}\right)$.

\section{Resultados numéricos}

Para avaliar o desempenho do algoritmo proposto foram realizadas simulações, no simulador [Drummond 2018], e comparação com o algoritmo Degraded [Wang et al. 2015], que considera degradação de banda passante mas não permite atraso na restauração. $O$ número de saltos foi usado para seleção de rota e First-Fit para atribuição de comprimento de onda. A topologia NSF (Fig. 1), com 14 nós e 42 enlaces de fibra unidirecionais (com 16 comprimentos de onda e capacidade de 10Gbps) foi usada na simulação. Cada nó possui 32 pares (input, output) de portas de agregação, sem capacidade de conversão de comprimento de onda. Assume-se que os nós 3, 4, 10, e 11 são nós DCs, cada um com 3000 unidades de armazenamento e 150 unidades de processamento. O número de unidades de armazenamento e processamento requisitadas para cada serviço é uniformemente distribuídos com valores médios de 100 e 5, respectivamente.

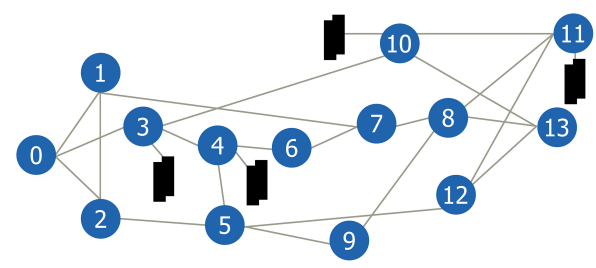

Figura 1. Topologia NSF.

Com intervalos de confiança de $95 \%$ de confiança, foram consideradas 10 execuções para cada ponto nos gráficos, cada execução envolvendo 10000 solicitações para estabelecimento de serviços na nuvem óptica. Estas solicitações são uniformemente 
distribuídas entre todos os pares de nós na rede. O tempo de duração de conexão e atraso na restauração seguem uma distribuição exponencial negativa com média de 60 unidades de tempo. A taxa de chegada de solicitações segue uma distribuição de Poisson, e as demandas de banda passante são distribuídas de acordo com a seguinte probabilidade: non-real-time (OC-12:5, OC-24:5, e $O C$-48:5); soft-real-time $(O C-12: 3, O C$-24:3, e $O C$-48:3); hard-real-time (OC-6:4, e $O C$-12:2). A carga em Erlangs é definida como a taxa média de chegada $\times$ a duração da chamada $\times$ a banda requisitada pelo serviço normalizada pela capacidade de um comprimento de onda. Falhas são uniformemente distribuídas entre todos os enlaces de fibra. $\mathrm{O}$ tempo entre falhas e o tempo de reparação são exponencialmente distribuídos com valores médios de 1000 e 10 unidades de tempo, respectivamente. Assume-se que enquanto um enlace está sob reparo nenhum outro enlace na rede pode falhar. As configurações utilizadas foram baseadas em [Wang et al. 2015].

As métricas usadas foram: $i$ ) número médio de serviços perdidos (interrompidos pela falha e não restaurados); ii) probabilidade de bloqueio de solicitações de serviço na nuvem (i.e., a percentagem de solicitações bloqueadas em relação ao número total de solicitações de serviço). Devido a limitação de espaço, somente estas duas métricas são apresentadas. Outros resultados podem ser encontrados em [Lisboa et al. 2017].

A Fig. 2 mostra o número de serviços perdidos em função da carga na rede. $\mathrm{O}$ número de serviços perdidos pelo algoritmo R3D é consideravelmente menor do que aquele gerado pelo algoritmo que não permite atraso na restauração (Degraded) para todas as cargas consideradas. Para carga de 30 Erlangs, 700 serviços foram interrompidos pela falha. Para este conjunto, o algoritmo R3D perdeu 55 serviços, enquanto o algoritmo Degraded perdeu 350 serviços. A maior diferença é verificada para a carga de 120 Erlangs, quando o R3D perde 210 serviços e o Degraded perde todos os serviços (1010) suspensos pela falha. A ideia central do algoritmo Degraded é realizar imediatamente a restauração de todos serviços interrompidos pela falha. Além disso, ele pode restaurar serviços das classes soft-real-time e non-real-time usando a metade da banda requisitada. A redução na banda previne a perda de serviços destas classes e mantém um nível mínimo aceitável de qualidade de serviço. Além da degradação de banda para os serviços soft-real-time e non-real-time, o algoritmo R3D pode postergar a restauração de serviços interrompidos da classe non-real-time e, consequentemente, este tipo de serviço tem um nova chance de reestabelecimento em um futuro próximo usando a banda liberada por outros serviços ou usando a banda de enlaces reparados. Estes resultados evidenciam que a tolerância ao atraso na restauração aumenta significativamente o número de serviços restaurados.

Na Fig. 3, apresenta-se a probabilidade de bloqueio de solicitações de serviço na nuvem em função da carga na rede. Tanto o R3D quanto o Degraded algoritmo produziram níveis aceitáveis de probabilidade de bloqueio. Ademais, o algoritmo proposto gerou alguma redução $( \pm 1,8 \%)$ no bloqueio comparado ao algoritmo Degraded.

\section{Conclusão}

Esta artigo apresentou um algoritmo (R3D) de restauração de serviços interrompidos por falha em um enlace da nuvem óptica. O R3D combina o uso de tolerância ao atraso na restauração e degradação de banda, os quais são determinados de acordo com a classe que um dado serviço pertence. Resultados numéricos mostram que nuvens ópticas podem se beneficiar do algoritmo proposto em relação a um maior número de serviços restaurados. 


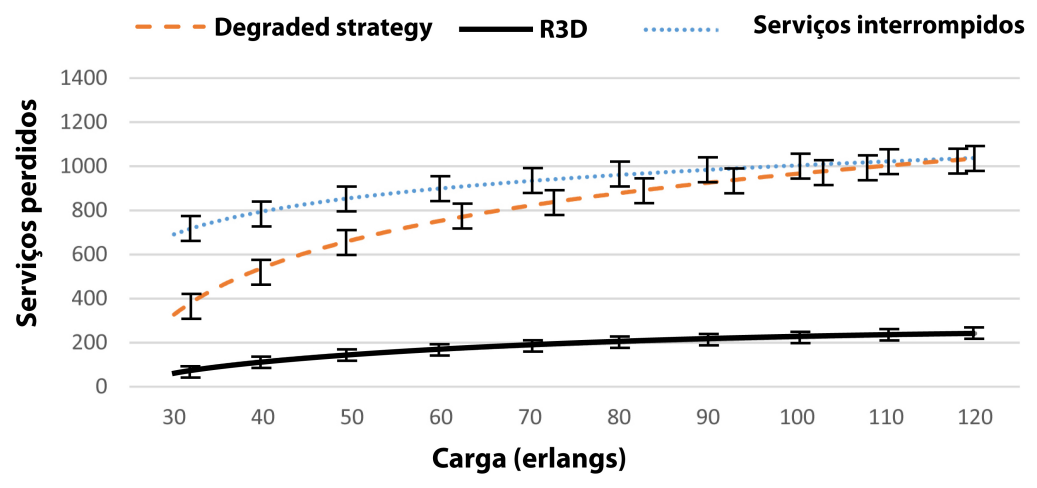

Figura 2. Número médio de serviços perdidos em função da carga na rede .

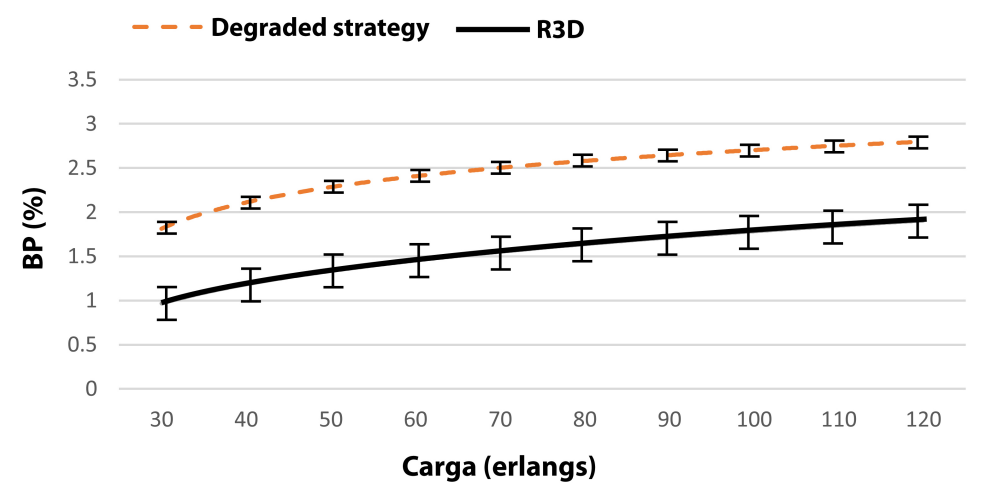

Figura 3. Probabilidade de bloqueio de solicitações de serviços $v s$ carga.

Além disso, o R3D não aumenta a probabilidade de bloqueio.

Sugere-se, como trabalho futuro, a investigação da degradação de serviços existentes, com tolerância ao atraso, para liberar recursos para serviços interrompidos que são sensíveis ao atraso. Indica-se, também, a investigação do impacto do estabelecimento de serviços com balanceamento de carga.

\section{Síntese das contribuições do trabalho}

As principais contribuições deste trabalho de Iniciação Científica são descritas a seguir:

- Introdução à leitura de artigos científicos e conceitos de simulação/ferramentas;

- Verificação do estado da arte para a recuperação de serviços em nuvens ópticas;

- Introdução da utilização conjunta de degradação de banda passante e tolerância ao atraso para a restauração de serviços em nuvens ópticas;

- Proposição de um algoritmo inédito, simples e de fácil implementação para a restauração de serviços em nuvens ópticas;

- Análise de complexidade computacional do algoritmo proposto;

- Introdução à análise de resultados de simulação e redação de artigos científicos;

- Desenvolvimento do trabalho de conclusão de curso (TCC) - defesa em 07/2017;

- Publicação na conferência internacional "IEEE Global Communications Conference" (GLOBECOM), Qualis A1 [Lisboa et al. 2017];

- O conhecimento alcançado durante a Iniciação Científica será a base para o mestrado iniciado no primeiro semestre de 2018. 


\section{Referências}

Bao, N. H., Tornatore, M., Martel, C. U., and Mukherjee, B. (2016). Fairness-aware degradation based multipath re-provisioning strategy for post-disaster telecom mesh networks. IEEE/OSA Journal of Optical Comm. and Networking, 8(6):441-450.

da Silva, C. N., Wosinska, L., Spadaro, S., Costa, J. C. W. A., Frances, C. R. L., and Monti, P. (2016). Restoration in optical cloud networks with relocation and services differentiation. IEEE/OSA Journal of Optical Comm. and Networking, 8(2):100-111.

Develder, C., Leenheer, M. D., Dhoedt, B., Pickavet, M., Colle, D., Turck, F. D., and Demeester, P. (2012). Optical networks for grid and cloud computing applications. Proceedings of the IEEE, 100(5):1149-1167.

Drummond, A. C. (2018). WDMSim - optical WDM networks simulator. http://www.lrc.ic.unicamp.br/wdmsim. Último acesso: 21-01-2018.

Ferdousi, S., Tornatore, M., Habib, M. F., and Mukherjee, B. (2015). Rapid data evacuation for large-scale disasters in optical cloud networks [invited]. IEEE/OSA Journal of Optical Communications and Networking, 7(12):B163-B172.

Habib, M. F., Tornatore, M., Dikbiyik, F., and Mukherjee, B. (2013). Disaster survivability in optical communication networks. Computer Communications, 36(6):630 - 644. Reliable Network-based Services.

Huang, S., Xia, M., Martel, C. U., and Mukherjee, B. (2010). A multistate multipath provisioning scheme for differentiated failures in telecom mesh networks. Journal of Lightwave Technology, 28(11):1585-1596.

Kachris, C. and Tomkos, I. (2012). A survey on optical interconnects for data centers. IEEE Communications Surveys Tutorials, 14(4):1021-1036.

Khabbaz, M. and Assi, C. (2015). Impact of job deadlines on the qos performance of cloud data centers. In IEEE Int. Conf. on Cloud Networking (CloudNet), pages 32-37.

Lisboa, F., Fonseca, K. V. O., Vieira, L. C., Monti, P., Figueiredo, G. B., and de Santi, J. (2017). Restoration Based on Bandwidth Degradation and Service Restoration Delay for Optical Cloud Networks. In IEEE Global Comm. Conf. (GLOBECOM), pages 1-6.

Natalino, C., Monti, P., França, L., Furdek, M., Wosinska, L., Francês, C. R., and Costa, J. W. (2015). Dimensioning optical clouds with shared-path shared-computing (spsc) protection. In IEEE 16th International Conference on High Performance Switching and Routing (HPSR), pages 1-6.

Wang, M., Furdek, M., Monti, P., and Wosinska, L. (2015). Restoration with service degradation and relocation in optical cloud networks. In Asia Communications and Photonics Conference 2015, page ASu5F.2. Optical Society of America.

Wang, W., Zhao, Y., Zhang, J., He, R., and Chen, H. (2016). Cross-stratum resource reservation (csrr) algorithm for deadline-driven applications in datacenter networks. Photonic Network Communications, 31(1):162-171.

Wang, Y., Su, S., Jiang, S., Zhang, Z., and Shuang, K. (2012). Optimal routing and bandwidth allocation for multiple inter-datacenter bulk data transfers. In IEEE International Conference on Communications (ICC), pages 5538-5542. 